\title{
Analyst
}

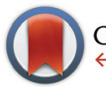

CrossMark

\&lick for updates

Cite this: Analyst, 2016, 141, 4562

Received 5th May 2016,

Accepted 17th June 2016

DOI: $10.1039 /$ c6an01044j

www.rsc.org/analyst

\section{Detection of prostate cancer using a voltammetric electronic tongue $\uparrow$}

\author{
Lluís Pascual, ${ }^{a}$ Inmaculada Campos, ${ }^{a}$ José-Luis Vivancos, ${ }^{\star a, b, c, d}$ Guillermo Quintás, ${ }^{\text {e,f }}$ \\ Alba Loras, ${ }^{d}$ M. Carmen Martínez-Bisbal, ${ }^{\text {a,c }}{ }^{2}$ Ramón Martínez-Máñez, ${ }^{a, c, d, g}$ \\ Francisco Boronat ${ }^{\mathrm{h}}$ and José Luis Ruiz-Cerdàd,h
}

\begin{abstract}
A simple method based on the multivariate analysis of data from urine using an electronic voltammetric tongue is used to detect patients with prostate cancer. A sensitivity of $91 \%$ and a specificity of $73 \%$ were obtained to distinguish the urine from cancer patients and the urine from non-cancer patients.
\end{abstract}

Prostate cancer (PCa) is the most frequent noncutaneous malignancy in the male population. In the United States it had an incidence of 238590 cases in 2013. ${ }^{1}$ Currently, testing the level of prostate specific antigen (PSA) in blood remains the most widely used procedure for PCa detection. When using a $4.0 \mathrm{ng} \mathrm{ml}^{-1}$ cutoff, the PSA test sensitivity and specificity are $34.9 \%$ and $63.1 \%$, respectively. ${ }^{2}$ However, a high level of serum PSA is not necessarily evidence of $\mathrm{PCa}^{3}$ and can also be caused by benign prostatic hyperplasia (BPH), inflammation of the prostatic gland, urinary retention or rectal palpation. ${ }^{4}$ Moreover, the PSA test can produce either false-negative results ${ }^{5}$ or over-diagnosis which leads to over-treatment of relatively indolent tumours. ${ }^{6}$ In addition, it is also worth mentioning that $\mathrm{BPH}$ is the most common benign tumor in men, with prevalence estimates ranging from $50 \%$ for men in their 50 s to $90 \%$ for men in their 90 s. $^{7}$ In this scenario, important studies have been conducted in the last decade to investigate new pro-

\footnotetext{
${ }^{a}$ Instituto Interuniversitario de Investigación de Reconocimiento Molecular y Desarrollo Tecnológico (IDM), Unidad Mixta Universitat Politècnica de València Universitat de València, Spain. E-mail: jvivanco@dpi.upv.es

${ }^{b}$ Departamento de Proyectos de Ingeniería, Universitat Politècnica de València, Camino de Vera s/n., 46022 Valencia, Spain

${ }^{c}$ CIBER de Bioingeniería, Biomateriales y Nanomedicina (CIBER-BNN), Spain. E-mail:mamarbis@upvnet.upv.es

${ }^{d}$ Unidad Mixta de Investigación en Nanomedicina y Sensores Universitat Politècnica de València, IIS La Fe de Valencia, Spain

${ }^{e}$ Leitat Technological Center, Safety and Sustainability Division, Valencia, Spain

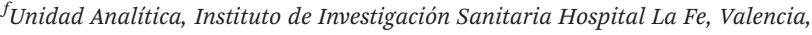
Spain

${ }^{g}$ Departamento de Quimica, Universitat Politècnica de València, Camino de Vera $s / n$., 46022 Valencia, Spain

${ }^{h}$ Servicio de Urología, Hospital Universitario y Politécnico La Fe, Valencia, Spain $\dagger$ Electronic supplementary information (ESI) available: Details of experimental data and data analysis. See DOI: 10.1039/c6an01044j
}

cedures to improve early PCa diagnosis and PCa risk prediction. ${ }^{8,9}$

From another point of view, it is well-known that neoplastic transformation is related to metabolic alterations which provides a key to differentiate tumours from normal tissues via the recognition of metabolic signatures in either tissues or biofluids. The metabolites of living cells are seen as the end products of the biological hierarchy, starting with activated genes (genome) and extending over the collection of gene transcripts (transcriptome) and proteins (proteome). Neoplastic transformation is considered to necessitate metabolic alterations to provide the bioenergetic and synthetic requirements of malignancy. ${ }^{10}$ It is on this basis that alterations to the metabolic signatures within biofluids or tissues reflect changes in phenotype and function and are the key to differentiate tumours from the normal tissues. ${ }^{11-13}$ Moreover, it is believed that metabolic alterations precede neoplastic proliferation. In this context, if such precancerous alterations are related to detectable metabolites, an early intervention may prevent cancer development or minimize neoplastic proliferation and invasion of local or distant structures. ${ }^{14}$

Following this approach, the changes in the levels of single metabolites and metabolite ratios have long been investigated as potential biomarker candidates for PCa. ${ }^{15}$ Nevertheless, in the particular case of $\mathrm{PCa}$, the current consensus suggests that there is not a single metabolite that can be directly related to the existence of PCa but it is the entire metabolic profile which is more sensitive in identifying and characterizing prostate cancer. ${ }^{16}$ According to this, the use of techniques that are prone to give a holistic picture of the samples is highly appealing and may find applications in PCa diagnosis. Most metabolomics studies have been reported on prostate in vivo and ex vivo in tissues and extracts, ${ }^{17-19}$ but studies on body fluids, especially urine, are scarce. ${ }^{19-21}$ The use of urine to detect PCa is clearly indicated since it contains products released directly from the prostate or carried within prostatic cells that are shed into urine. ${ }^{22}$ Moreover, urine is collected non-invasively and requires minimal sample preparation prior to the analysis. ${ }^{23}$ 
From another field, electronic tongues (e-tongues) have been introduced as a suitable tool applied to characterize the attributes of complex systems. ${ }^{24}$ E-tongues are based on unspecific sensors usually integrated in an array, and their response is commonly analysed by using suitable pattern recognition algorithms. $^{24}$ The idea of this technique is that although the specificity of each sensor is low, the combination of several unspecific sensors entails a very large information potential. Particularly voltammetric e-tongues have been reported to be versatile, simple, robust and highly sensitive to changes in the redox attributes of the samples. E-tongues have found a number of applications in the classification of complex systems such as food and water samples. ${ }^{25}$ However the use of e-tongues for cancer detection in biofluids is much less common. A potentiometric e-tongue has been applied to study urine samples from bladder cancer patients (17 tumors, and 10 controls). ${ }^{26}$ However, preliminary results using PCA for an unsupervised analysis did not include an estimate of the predictive performance of the system. Moreover, potentiometric e-tongues have also been used to determine urea and creatinine in urine $\mathrm{u}^{27}$ and to detect urinary system dysfunctions and creatinine levels. ${ }^{26}$ However, as far as we know, voltammetric e-tongues have not been applied to the study of urine to detect PCa.

Voltammetric e-tongues are expected to respond to the presence of electrochemically-active species. In this context it has been found from basic research studies that during tumour growth, protein changes in malignant cells lead to peroxidation of the cell membrane components. It has also been reported that the levels of glutathione (a naturally occurring reducing agent in cells) increase for some diseases such as cancer. ${ }^{28}$ Moreover certain types of cancer cells are known to produce substantial amounts of reactive oxygen species (ROS). ${ }^{29}$ Prostate cells have a distinct metabolic profile reflecting the production of citrate, PSA and polyamines (spermine and myo-inositol), ${ }^{30}$ and citrate, which is a redox-active molecule, is a marker of normal prostate cells. ${ }^{30}$ Moreover, prostate is known to contain very high intracellular zinc levels. ${ }^{30}$ However, when prostate cells undergo neoplastic transformation they have been reported to lose the capacity to accumulate zinc and citrate. ${ }^{30}$ These and other similar changes, which are envisioned to occur during prostate tumour growth, may provide an "electrochemical" fingerprint for $\mathrm{PCa}$ in urine samples that might be detected by using an e-tongue.

Based on these concepts, we report herein a study on the potential use of a simple voltammetric e-tongue based on metallic electrodes for the detection of PCa from urine samples.

Urine samples were obtained from Caucasian patients recruited in the urological service in Hospital Universitario y Politécnico La Fe (Valencia, Spain). A total of 114 samples were studied using an e-tongue, 71 urine samples from patients with PCa pre-surgery, 26 urine samples from patients with PCa post-surgery, and 17 urine samples from patients with BPH (22 $+17=43$ urine samples without cancer) as shown in Table 1 . The set of patients with PCa contained both patients with tumour subsidiaries of active treatment (radical prostatectomy
Table 1 Patient and control samples considered in the study

\begin{tabular}{llllll}
\hline Group & Number & Perc. \% & Control type & Number & Perc. \% \\
\hline PCa & 71 & 62.3 & & & 62.3 \\
Control & 43 & 37.7 & Post-prostatectomy & 26 & 22.8 \\
& & & HBP & 17 & 14.9
\end{tabular}

or radiotherapy) and patients with tumours that fulfilled the criteria of indolent tumours (only 1 or 2 biopsy cores invaded by prostate cancer, Gleason score $<6$, PSA-level at diagnosis $\leq 10 \mathrm{ng} \mathrm{mL} \mathrm{m}^{-1}$, clinical stage $\mathrm{T} 1 \mathrm{c}$ or $\mathrm{T} 2$ ). The patients of this later subset were left untreated and included in an active surveillance program. Table 2 shows the age, PSA, cT, Gleason score, risk group and the final treatment decision for the PCa patients included in the study.

When collected, the urine samples were frozen and stored at $-80{ }^{\circ} \mathrm{C}$ until analysed. When unfrozen the samples were centrifuged at $2500 \mathrm{rpm}$ for 5 minutes to eliminate solids and other insoluble materials and then aliquoted. All the samples were thawed to room temperature (approximately $21^{\circ} \mathrm{C}$ ) prior to analysis. The samples were managed (processed, preserved and supplied) by Biobanco La Fe, authorized and certified biobank according to the requisites in Real Decreto 1716/2011 (ref. PT13/0010/0026), and Standard ISO 9001:2008.

The e-tongue used in this study was an array of seven working electrodes ( $\mathrm{Ir}, \mathrm{Rh}, \mathrm{Pt}, \mathrm{Au}, \mathrm{Ag}$, $\mathrm{Co}$ and $\mathrm{Cu}$ ) with a purity of $99.99 \%$ and a $2-\mathrm{mm}$ diameter (GoodFellow). The set of electrodes was formed by metal wire electrodes housed inside a stainless steel cylinder that was used as the body of the electronic tongue system and as the counter electrode. As the

Table 2 Age and clinical data of patients with PCa $(n=71)$

\begin{tabular}{|c|c|c|c|}
\hline Clinical data & Range & Number & Percentage \% \\
\hline \multirow[t]{2}{*}{ Age (years) } & $50-65$ & 33 & 46.5 \\
\hline & $66-80$ & 38 & 53.5 \\
\hline \multirow[t]{3}{*}{$\operatorname{PSA}\left(\mathrm{ng} \mathrm{ml}^{-1}\right)$} & $<5$ & 14 & 19.7 \\
\hline & $6-10$ & 44 & 62.0 \\
\hline & $10-20$ & 13 & 18.3 \\
\hline \multirow[t]{4}{*}{$\mathrm{cT}^{a}$} & cT1c & 21 & 29.6 \\
\hline & $\mathrm{cT} 2 \mathrm{~b}$ & 1 & 1.4 \\
\hline & $\mathrm{cT} 2 \mathrm{c}$ & 47 & 66.2 \\
\hline & cT3b & 2 & 2.8 \\
\hline \multirow[t]{3}{*}{ Gleason score $^{b}$} & $<6$ & 42 & 59.2 \\
\hline & 7 & 18 & 25.4 \\
\hline & $8-10$ & 11 & 15.4 \\
\hline \multirow[t]{3}{*}{ Risk group $^{c}$} & Low & 42 & 59.2 \\
\hline & Intermediate & 17 & 23.9 \\
\hline & High & 12 & 16.9 \\
\hline \multirow[t]{4}{*}{ Treatment } & Active surveillance & 22 & 31.0 \\
\hline & Hormonal therapy & 2 & 2.8 \\
\hline & Radiotherapy & 13 & 18.3 \\
\hline & Radical prostatectomy & 34 & 47.9 \\
\hline
\end{tabular}

${ }^{a} \mathrm{cT}$ is a PCa staging related to the extension and location of the tumour. ${ }^{b}$ Gleason score is based on the histological examination of the biopsy. It ranges from 2 to 10, with 2 representing the most welldifferentiated tumours and 10 the least-differentiated tumours. ${ }^{c}$ Risk group low means cT2abc and Gleason $\leq 6$, intermediate means cT2abc and Gleason $=7$, and high risk means cT3ab or Gleason 8-10. 
reference electrode, a saturated calomel electrode was used. The e-tongue was controlled with a home-made software application that runs on a PC and home-made electronic equipment. $^{31}$ A Large Amplitude Pulse Voltammetry (LAPV) waveform $^{24}$ was applied to all the seven working electrodes in contact with the urine sample and the resulting current $v$. time profile for each electrode was measured. The LAPV composed of 42 pulses of $30 \mathrm{~ms}$ ranging from -1000 to $1000 \mathrm{mV}$ for noble metals, and -500 to $500 \mathrm{mV}$ for non-noble metals (see the ESI $\dagger$ for details). Fig. 1A shows the LAPV waveform. The LAPV waveform was applied five times to each urine sample.

Finally, the mean of the five records was normalized using the second pulse which is related to the conductivity of the solution. This procedure minimizes the response of the e-tongue to variations in salt concentrations in the different urine samples. The presence of strong electrolytes in the urine as inorganic salts might have masked the information of $\mathrm{PCa}$ related substances. As an example of the response found, Fig. 1B shows the current/time response obtained for the $\mathrm{Ag}$

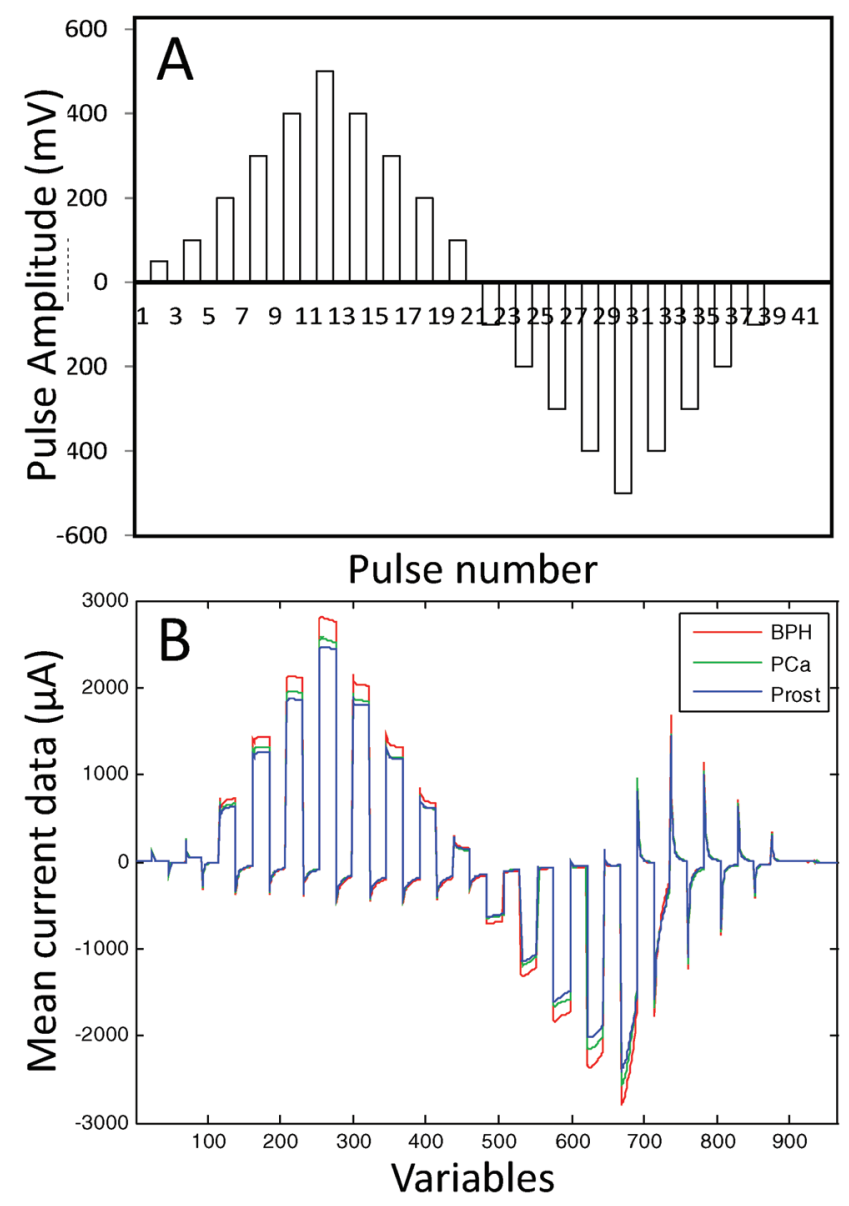

Fig. 1 (A) The pulse sequence applied and (B) the current/time response obtained for a certain electrode $(\mathrm{Ag})$ for three different urine samples from a patient with PCa (PCa), a PCa patient after radical prostatectomy (Prost) and a patient diagnosed with benign prostatic hyperplasia (BPH). electrode in three different urine samples from a patient with $\mathrm{PCa}$, a PCa patient after radical prostatectomy and a patient diagnosed with $\mathrm{BPH}$.

Data analysis was performed using MATLAB 2014a (The Mathworks), and PLS_Toolbox (version: 7.9.3). A schematic work flow of the experimental approach for the data analysis is described in Fig. 2. The 114 samples were randomly split into sets for calibration (66\%) and sets for cross-validation (33\%). In all cases, the randomly selected calibration sets included a total of 76 samples containing 47 patients with $\mathrm{PCa}$ and 29 control samples (17 after radical prostatectomy and 12 diagnosed with $\mathrm{BPH}$ ). Besides the cross-validation sets included 38 samples containing 24 patients with PCa and 14 control samples ( 9 after radical prostatectomy and 5 with $\mathrm{BPH}$ ).

From the data collected, studies were performed to evaluate the best selection of electrodes using a two-level full factorial design for 7 electrodes obtaining 127 experiments or combination of electrodes.

The initial PLSDA figures of merit for the different combinations of electrodes were obtained after 12 iterations of a random 8-fold. The calibration set was used for model development. Supervised discriminant analysis was performed using partial least squares (PLSDA) and a maximum number of 7 latent variables (LVs). The X-block (i.e. data of combination of electrodes) was auto-scaled and the $y$ vector containing class labels (i.e. -1 and +1 for control and PCa samples, respectively) was mean centred. From the model 4 LVs were retained. In order to avoid overoptimistic results, the statistical significance of the model was evaluated using cross-validation. To ensure the representativeness of the results, the random dataset division was repeated 500 times, and the number of misclassifications was used for the selection of the best combination of electrodes (vide infra).

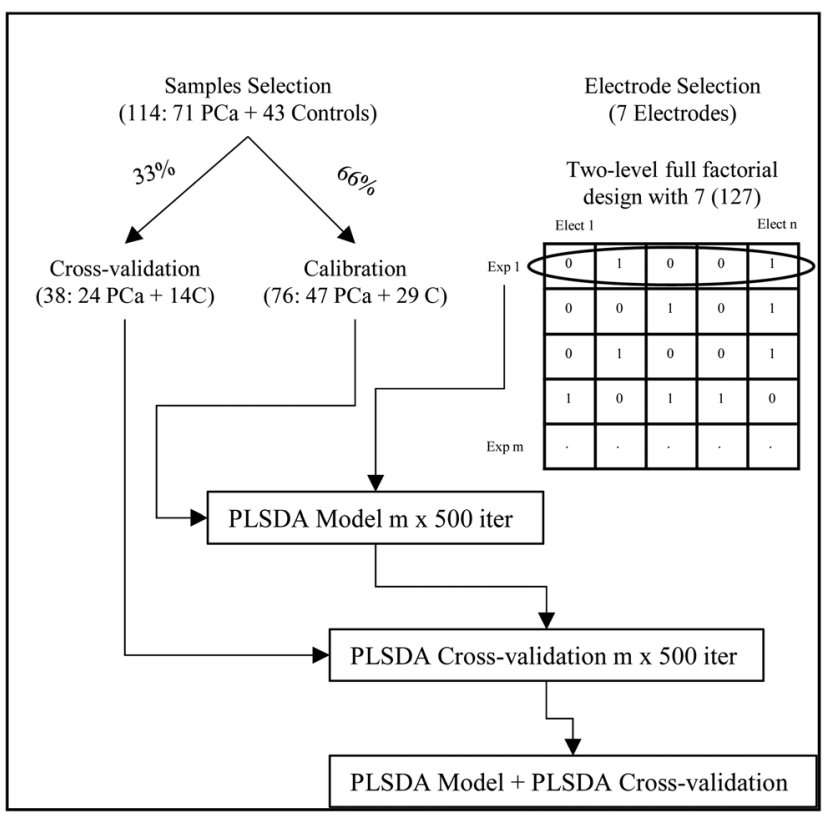

Fig. 2 Schematic work flow of the experimental approach. 
Fig. 3 depicts the best selection of each combination of electrodes (using 1 to 7 electrodes) represented by a boxplot and the number of misclassifications in the cross-validation sets. The best results were observed when using data from three (Ir, Au, Ag), four (Ir, Pt, Au, Ag) and five (Ir, Rh, Pt, Au, $\mathrm{Ag}$ ) electrodes. For instance, as shown in Fig. 3, when data from Ir, Au and Ag electrodes (combination C in Fig. 3) were used to predict $\mathrm{PCa}$ in 500 randomly cross-validated subsets, the median of misclassifications was 7 out of 38 , and in $75 \%$ of the cases (in 375 out of 500 cross-validated subsets) misclassifications were equal or lower than 9. The simple use of one (Pt, combination A in Fig. 3) or two (Ir, Rh, combination B in Fig. 3) electrodes resulted in a worse classification indicating that it is the combination of the data of several electrodes that led to a better sorting of the samples. When six or seven electrodes were used (see Fig. 3 combinations $\mathrm{F}$ and $\mathrm{G}$ respectively) the number of misclassifications also increased. Fig. 3 also shows that the combination of 4 and 5 electrodes (combinations D and E, respectively) gave a number of similar misclassified samples than the combination of 3 electrodes. On the basis of these results the combination of 3 electrodes (i.e. Ir, $\mathrm{Au}$ and $\mathrm{Ag}, \mathrm{C}$ in Fig. 3) was selected, being the simplest combination of metals with the better results (a smaller number of metal electrodes for the same misclassified samples). This combination of three electrodes was used for the calculation of a second PLSDA model (see the ESI† for calculations on models D and E).

In this second PLSDA model three LVs were used based on the results obtained after 12 iterations of a random 8-fold (see the ESI $\uparrow$ for details). To avoid overoptimistic results, the statistical significance of the model was evaluated using the external validation set which consisted of a total of 37 urine samples (22 PCa urine samples and 15 control urine samples).

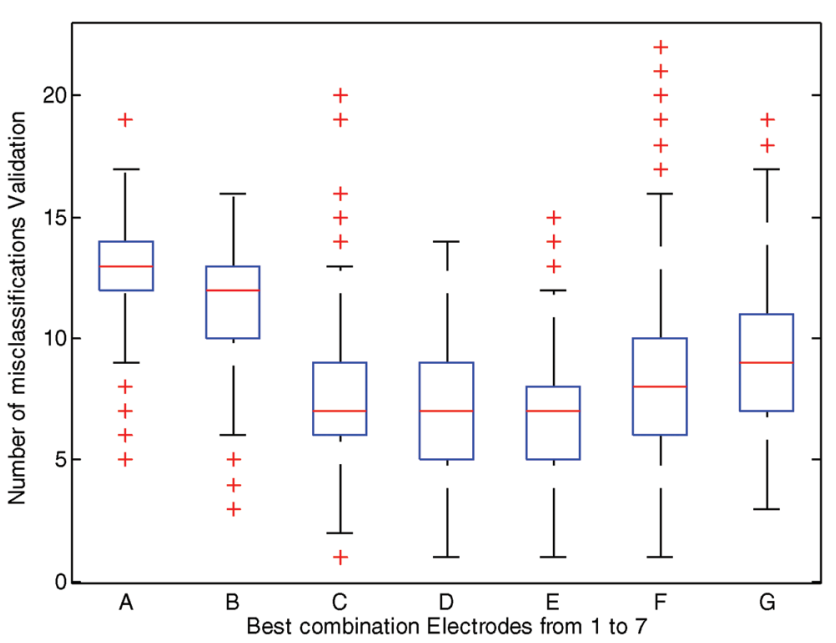

Fig. 3 Boxplot of the best selection for each combination of electrodes in terms of the number of misclassifications after 500 iterations in the cross-validation set (4 LVs). (A) Ag, (B) Ir, Rh, (C) Ir, Au, Ag, (D) Ir, Pt, Au, $\mathrm{Ag},(\mathrm{E}) \mathrm{Ir}, \mathrm{Rh}, \mathrm{Pt}, \mathrm{Au}, \mathrm{Ag},(\mathrm{F}) \mathrm{Ir}, \mathrm{Rh}, \mathrm{Pt}, \mathrm{Au}, \mathrm{Ag}, \mathrm{Cu},(\mathrm{G}) \mathrm{Ir}, \mathrm{Rh}, \mathrm{Pt}, \mathrm{Au}, \mathrm{Ag}, \mathrm{Co}$, $\mathrm{Cu}$.
The results are summarized in Table 3, whereas the predicted values are also shown in Fig. 4.

In this testing phase the use of data from the combination of $\mathrm{Ir}, \mathrm{Au}$ and $\mathrm{Ag}$ electrodes in urine samples was able to correctly classify 20 samples out of 22 for the patients with PCa, whereas it classified 11 samples out of 15 correctly from control patients. This resulted in a sensitivity of $91 \%$ and a specificity of $73 \%$.

Positive predictive values were 0.833 with lower 0.626 and upper 0.953 (estimation at 95\%). Negative predictive values were 0.846 with lower 0.546 and upper 0.981 (estimation at 95\%). Moreover, a revision of the misclassified data indicated that the two samples that were predicted as false negatives in Table 3 belonged to patients with indolent tumours and if these data are excluded in the validation set, a sensitivity of $100 \%$ was reached.

The scientific basis of this ability of e-tongues to detect a signature of PCa is likely linked to the presence of certain substances that are released either directly into the urine or carried within prostatic cells that are shed into urine. Besides, these preliminary data might reflect the existence of a potential "electrochemical" signature of $\mathrm{PCa}$ in urine samples.

Table 3 Calibration and validation results obtained by PLSDA using Ir, Au and Ag electrodes and 3 LVs. 74 for calibration and 37 for validation

\begin{tabular}{lrrrrr} 
& \multicolumn{2}{c}{ Calibration } & & \multicolumn{2}{c}{ Validation } \\
\cline { 2 - 3 } \cline { 5 - 6 } \cline { 5 - 6 } & PCa & Control & & PCa & Control \\
\hline Predicted as PCa & 41 & 0 & & 20 & 4 \\
Predicted as control & 5 & 28 & & 2 & 11 \\
& 46 & 28 & & 22 & 15
\end{tabular}

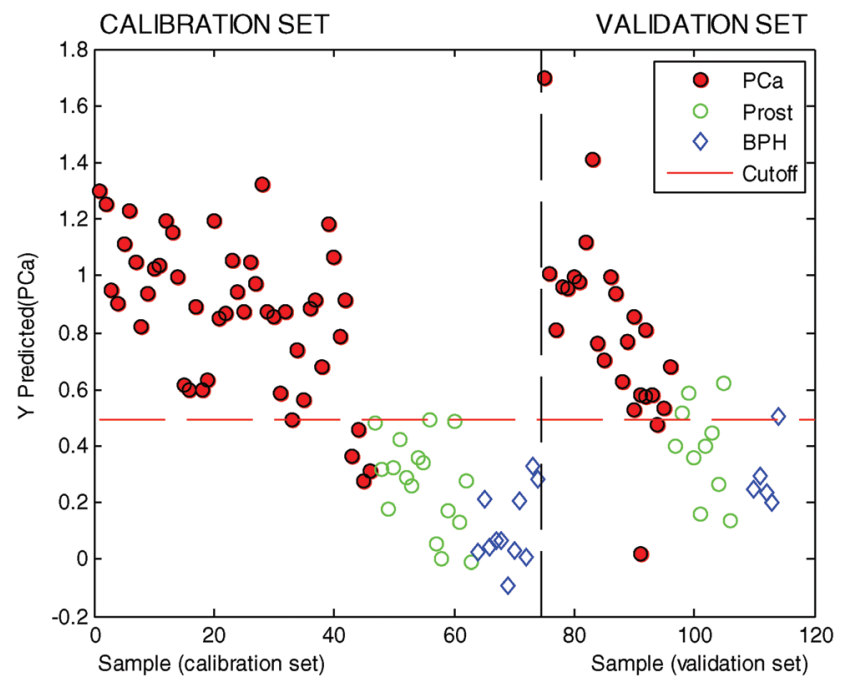

Fig. 4 Prediction of calibration and validation datasets (3 LVs) using Ir, $\mathrm{Au}$ and $\mathrm{Ag}$ electrodes. Split into calibration and validation using the Kennard-Stone method at $66 \%$. The model has been created using 2 classes (PCa and control) only for visualization purpose. Samples from patients after radical prostatectomy (Prost) and BPH patients are shown. 
However these electroactive molecules remain unknown for the moment and should be assessed by specific HPLC-MS and NMR analysis which are currently being performed.

\section{Conclusions}

PCa detection has been pursued using a large variety of techniques and studying different kinds of samples such as tumour tissue extracts and different body fluids. Although these studies have increased the biological understanding of PCa, they usually fail in providing a picture of the malignancy status. The current agreement is that entire metabolic profiles are more sensitive in identifying PCa rather than the changes of a unique metabolite.

We have shown here that after training, a simple e-tongue based on metallic electrodes was able to distinguish urine samples from patients with prostate cancer PCa from noncancer urine samples (patients after radical prostatectomy and patients diagnosed with $\mathrm{BPH}$ ) with a sensitivity of $91 \%$ and a specificity of $73 \%$. In particular the e-tongue correctly classified 20 samples out of 22 for patients with $\mathrm{PCa}$, whereas it classified 11 samples out of 15 correctly from non-cancer urine samples. Moreover, it is also noteworthy that potential biases that could have arisen from the presence of other diseases or particular food and drink consumption were not explored in our protocol but despite this lack of information, the e-tongue was able to overcome these potential confounding factors. This might rely on the detection of changes in the "electrochemical profile" informative of a pathological condition, better than reporting the changes of single metabolites that might be implied in diverse pathologies. The specificity and sensitivity obtained by the e-tongue in urine is higher compared to the PSA test in blood which is the most widely used procedure for $\mathrm{PCa}$ detection. The results we have obtained are of interest and point towards the potential use of e-tongues in the identification of patients with PCa using low cost equipment and a simple and fast analysis procedure with voltammetric e-tongues in urine, which is a body fluid easy to obtain and in a non-invasive way.

Despite the fact that the roadmap from these results to an extended use of the e-tongue test in clinical practice remains long and uncertain, given the relatively low cost of e-tongues and the potential automatization of sample measurement and multivariate statistical analysis, the proof of principle presented here suggests that this simple approach might have great potential for clinical applications for both PCa diagnosis and simple monitoring of patients after therapy.

The authors gratefully acknowledge the Ministerio de Economía y Competitividad and FEDER (project MAT201564139-C4-1-R (MINECO/FEDER)), the Generalitat Valenciana (project PROMETEOII/2014/047) and CIBER-BBN (NANOPROBE project) for their financial support. A. L. is grateful to the Generalitat Valenciana for her grant (Vali+d ACIF: 2015/115).

\section{Notes and references}

1 R. Siegel, D. Naishadham and A. Jemal, CA-Cancer J. Clin., 2013, 63, 11-30.

2 E. D. Crawford, S. Leewansangtong, S. Goktas, K. Holthaus and M. Baier, Prostate, 1999, 38, 296-302.

3 P. F. Pinsky, B. S. Kramer, E. D. Crawford, R. L. Grubb, D. A. Urban, G. L. Andriole, D. Chia, D. L. Levin and J. K. Gohagan, Urology, 2016, 68, 352-356.

4 T. G. Armitage, E. H. Cooper, D. W. W. Newling, M. R. G. Robinson and I. Appleyard, Br. J. Urol., 1988, 62, 584-589.

5 M. Törnblom, U. Norming, J. Adolfsson, C. Becker, P.-A. Abrahamsson, H. Lilja and O. Gustafsson, Urology, 1999, 53, 945-950.

6 C. H. Bangma, S. Roemeling and F. H. Schröder, World J. Urol., 2007, 25, 3-9.

7 H. Lepor, Rev. Urol., 2004, 6, S3-S10.

8 W. J. Catalona, A. V. D'Amico, W. F. Fitzgibbons, O. Kosoko-Lasaki, S. W. Leslie, H. T. Lynch, J. W. Moul, M. S. Rendell and P. C. Walsh, Ann. Intern. Med., 2012, 157, 137.

9 V. A. Moyer, Ann. Intern. Med., 2012, 157, 120.

10 L. C. Costello and R. B. Franklin, Mol. Cancer, 2006, 5, 17.

11 J. L. Griffin and J. P. Shockcor, Nat. Rev. Cancer, 2004, 4, 551-561.

12 C. Abate-Shen and M. M. Shen, Nature, 2009, 457, 799-800.

13 J. L. Spratlin, N. J. Serkova and S. G. Eckhardt, Clin. Cancer Res., 2009, 15, 431-440.

14 M. J. Roberts, H. J. Schirra, M. F. Lavin and R. A. Gardiner, Korean J. Urol., 2011, 52, 79-89.

15 L. L. Cheng, M. A. Burns, J. L. Taylor, W. He, E. F. Halpern, W. S. McDougal and C. L. Wu, Cancer Res., 2005, 65, 30303034.

16 E.-M. Spur, E. A. Decelle and L. L. Cheng, Eur. J. Nucl. Med. Mol. Imaging, 2013, 40, 60-71.

17 T. Kobus, A. J. Wright, E. Weiland, A. Heerschap and T. W. J. Scheenen, Magn. Reson. Med., 2015, 73, 1-12.

18 E. A. Decelle and L. L. Cheng, NMR Biomed., 2014, 27, 9099.

19 A. Sreekumar, L. M. Poisson, T. M. Rajendiran, A. P. Khan, Q. Cao, J. Yu, B. Laxman, R. Mehra, R. J. Lonigro, Y. Li, M. K. Nyati, A. Ahsan, S. Kalyana-sundaram, B. Han, X. Cao, J. Byun, G. S. Omenn, D. Ghosh, S. Pennathur, D. C. Alexander, A. Berger, J. R. Shuster and J. T. Wei, Nature, 2009, 457, 910-914.

20 J.-N. Cornu, G. Cancel-Tassin, V. Ondet, C. Girardet and O. Cussenot, Eur. Urol., 2011, 59, 197-201.

21 P. Zaragozá, J. L. Ruiz-Cerdá, G. Quintás, S. Gil, A. M. Costero, Z. León, J.-L. Vivancos and R. MartínezMáñez, Analyst, 2014, 139, 3875-3878.

22 R. R. Drake, K. Y. White, T. W. Fuller, E. Igwe, M. A. Clements, J. O. Nyalwidhe, R. W. Given, R. S. Lance and O. J. Semmes, J. Proteomics, 2009, 72, 907-917.

23 M. Truong, B. Yang and D. Jarrard, J. Urol., 2013, 189, 422-429. 
24 F. Winquist, Microchim. Acta, 2008, 163, 3-10.

25 A. Riul, C. a. R. Dantas, C. M. Miyazaki and O. N. Oliveira, Analyst, 2010, 135, 2481-2495.

26 L. Lvova, E. Martinelli, F. Dini, A. Bergamini, R. Paolesse, C. Di Natale and A. D’Amico, Talanta, 2009, 77, 10971104.

27 M. Gutiérrez, S. Alegret and M. del Valle, Biosens. Bioelectron., 2007, 22, 2171-2178.
28 N. Traverso, R. Ricciarelli, M. Nitti, B. Marengo, A. L. Furfaro, M. A. Pronzato, U. M. Marinari and C. Domenicotti, Oxid. Med. Cell. Longevity, 2013, 2013, 972913.

29 W. Dröge, Physiol. Rev., 2002, 82, 47-95.

30 B. J. Trock, Urol. Oncol., 2011, 29, 572-581.

31 I. Campos, M. Alcañiz, R. Masot, J. Soto, R. MartínezMáñez, J. L. Vivancos and L. Gil, Sens. Actuators, B, 2012, 161, 556-563. 